\title{
Endovenöse Behandlung der Stamm- und Seitenastvarikosis
}

Anina Lukhaup

\begin{abstract}
Mit den modernen endovenösen Therapieverfahren kann eine Stamm- und Seitenastvarikosis schonend und effektiv behandelt werden. Das in Deutschland am längsten zugelassene und am häufigsten angewandte Verfahren ist die segmentale Radiofrequenzablation. Erfahren Sie hier am Beispiel einer Radiowellenbehandlung der Vena saphena magna mit Schaumsklerosierung der Seitenastvarikosis, wie die endovenöse Behandlung Schritt für Schritt abläuft.
\end{abstract}

Endovenöse Verfahren I Ein Krampfaderleiden wird - abhängig vom Krankheitsstadium und der Beschwerdesymptomatik - entweder konservativ, klassisch operativ oder endovenös behandelt. Endovenöse Verfahren unterteilen sich in hitzeabhängige und -unabhängige Methoden. Zu ersteren zählen

- die hier vorgestellte segmentale Radiofrequenzablation,

- die endovenöse Laserablation (ELVeS),

- radiofrequenzinduzierte Thermotherapie $\left(\right.$ RFIT $\left.^{\circledR}\right)$ sowie

- Heissdampf(SVS, steam vein sklerosis).

Nicht-thermische Therapiealternativen sind die mechanochemische Ablation mit ClariVein ${ }^{\circledR}$ Katheter, eine Behandlung mittels CyanoacrylatGewebekleber (Venaseal-Closure ${ }^{\circledR}$ ) sowie die Schaumsklerosierung mit Aetoxysklerol ${ }^{\circledR}$.

Schon lange etabliert | Die segmentale Radiofrequenzablation wird in Deutschland bereits seit 18 Jahren durchgeführt und ist damit das endo-

Abb. 1 Punktionsnadel, Schleuse (7F) mit Führungsdraht, Skalpell, $10 \mathrm{ml}$ - und $5 \mathrm{ml}$ Spritzen, Lokalanästhetikum, Infiltrationsnadel zur Applikation der Tumeszenzanästhesie, Verlängerungsschlauch zur Tumeszenzpumpe, Kochsalzlösung zum Durchspülen des Katheters, Ultraschallgel, Ultraschallsondenüberzug, sterile Abdecktücher.

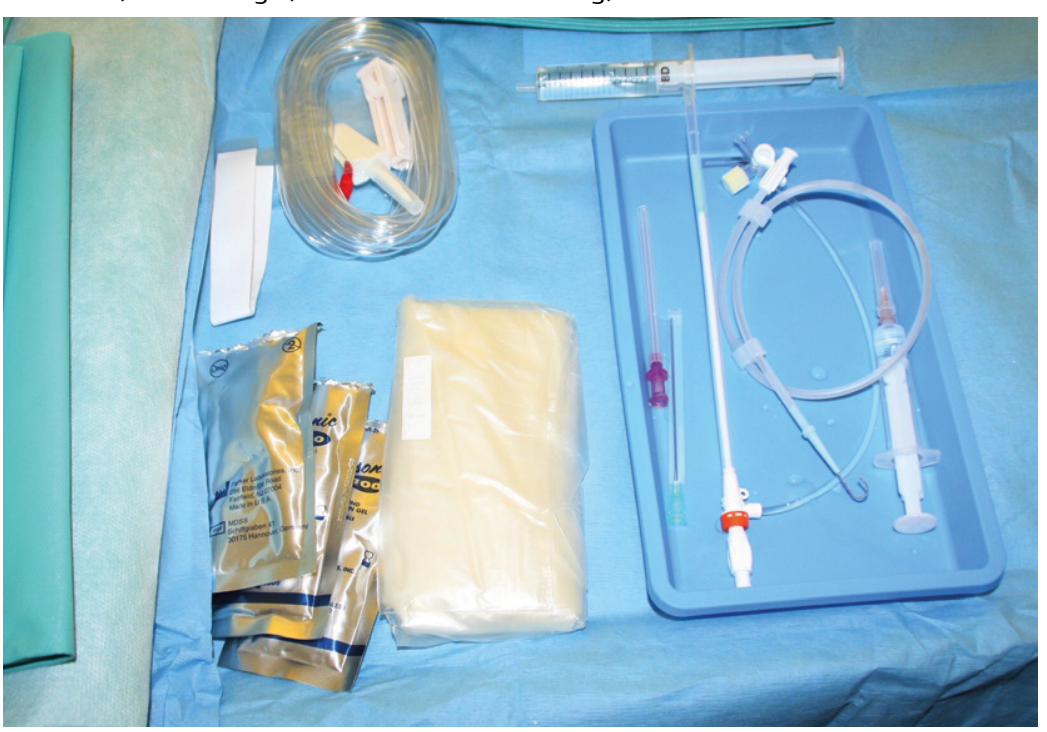

venöse Therapieverfahren mit der längsten $\mathrm{Zu}$ lassung und vermutlich den größten Behandlungszahlen $[1,2]$.

Durch die Kombination mit Schaumsklerosierung der Seitenastvarikosis kann in vielen Fällen auf eine chirurgische Venenentfernung verzichtet werden.

Was passiert bei der Radiowellenablation? I An der Spitze des Venefit ${ }^{\circledR}$-Katheters befindet sich ein $7 \mathrm{~cm}$-langes Heizelement. Über jeweils $20 \mathrm{Se}-$ kunden wird die Venenwand auf $120^{\circ} \mathrm{C}$ erhitzt und so geschädigt. Nach Behandlung eines GefäßAbschnitts wird der Katheter um jeweils $6,5 \mathrm{~cm}$ (Kathetermarkierung) zurückgezogen und das weiter distal gelegenen Segment behandelt. Crossennah und auch bei sehr großem Gefäß- Durchmesser ist eine weitere Impulsabgabe notwendig, um einen sicheren Gefäßverschluss zu erreichen. Ein kürzeres Heizelement zur Behandlung kurzstreckiger Venenabschnitte ist verfügbar $(3 \mathrm{~cm})$.

Voraussetzungen | Für die Behandlung sind gute Kenntnisse der Farbduplexsonografie, der möglichen Komplikationen sowie deren leitliniengerechtes Management vorausgesetzt. Ein Qualitätsmanagement ist empfehlenswert [2]. Dieses wird zum Beispiel über die deutsche Gesellschaft für Phlebologie zur Verfügung gestellt [3].

Anamnese I Diese konzentriert sich auf:

- Art und Dauer der varizenbedingten Beschwerdesymptomatik

- Thrombophlebitiden oder Venenthrombosen in der Vorgeschichte

- bisherige Veneneingriffe

- weitere Vorerkrankungen und Medikation

Die Familienanamnese bzgl. Varikosis und thromboembolischer Ereignisse ist ebenfalls wichtig.

Planung des Eingriffs I Mit dem detaillierten farbduplexsonografischen Befund (Stadien-Einteilung nach Hach, distaler Insuffizienzpunkt, Gefäß-Durchmesser, anatomische Besonderheiten, 


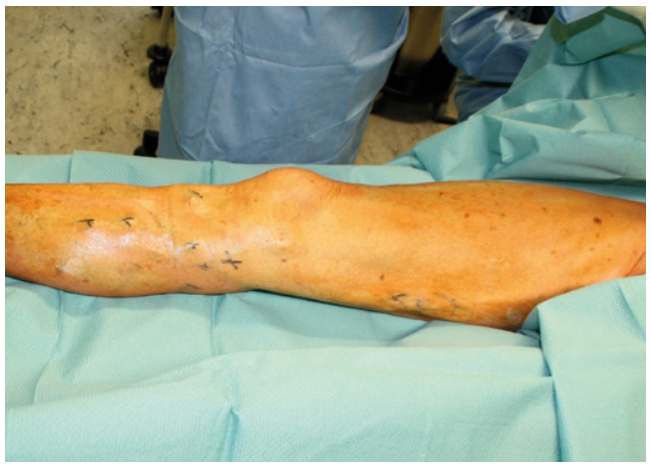

Abb. 2 Die vorab im Stehen angezeichneten Gefäßmarkierungen erleichtern das Auffinden der Punktionsstellen der Stammvene sowie der Seitenäste.

Hinweis auf abgelaufenen Phlebitis) wird Art und Umfang des Eingriffs geplant (ein-/beidseitig, zusätzliche Sklerosierung oder Exhairese).

Aufklärung | Mindestens 24 Stunden vor dem Eingriff wird der Patient mit schriftlicher Dokumentation aufgeklärt. In Abhängigkeit des individuellen Risikos führen wir eine Laboruntersuchung und EKG durch. Mögliche Komplikationen sind:

- hitzeinduzierte Nervenläsion mit resultierender Parästhesie

- oberflächliche Venenentzündung

- Thrombose

- Hyperpigmentierung

Insbesondere bei der Sklerosierungsbehandlung kommt es nicht selten zu einer Hyperpigmentierung im Gefäßverlauf. Dieser medizinische harmlose, aber u. U. kosmetisch störende Befund bildet sich i. d. R. nach einigen Wochen zurück. Eine explizite Patienteninformation und Aufklärung ist hier wichtig [4].

\section{Indikation}

- Stamm- und Seitenastvarikosis

- Rezidiv-Befunde nach Stripping-OP der Stammvenen (für thermische Verfahren in der Regel intrafasziale Gefäß-Abschnitte) [1].

\section{Absolute Kontraindikation}

- akute tiefe Becken-Beinvenenthrombose

- akute Thrombo- oder Varikophlebitis

- pAVK Stadium III und IV nach Fontaine

\section{Relative Kontraindikation}

- schwere Allgemeinerkrankung ASA $\geq 4$

- pAVK IIb nach Fontaine

- Schwangerschaft

- Thrombophilie

- Lymphödem

bei zusätzlicher Schaumsklerosierung:

- bekanntes symptomatisches offenes Foramen ovale

- Unverträglichkeit gegen Sklerosierungsmittel [5]

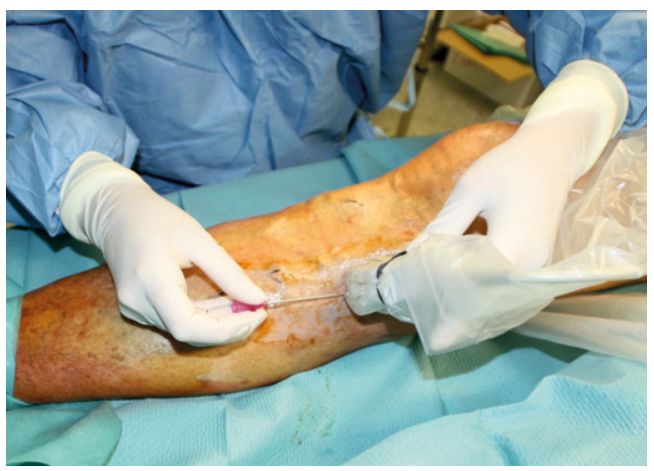

Abb. 3 Die Punktionsnadel wird mittig nah am Schallkopf aufgesetzt und unter Ultraschallsicht vorgeschoben.

Material | Benötigt werden Generator, Tumeszenzpumpe mit Fußpedal, Tumeszenzlösung sowie ein Kipptisch.

Steriles Material | Venefit-Katheter, Punktionskanüle, Schleuse (7F) mit Führungsdraht, Skalpell, $10 \mathrm{ml}$ und $5 \mathrm{ml}$ Spritzen, Lokalanästhetikum, Infiltrationsnadel zur Applikation der Tumeszenzanästhesie, Verlängerungsschlauch zur Tumeszenzpumpe, Kochsalzlösung zum Durchspülen des Katheters, Ultraschallgel, steriler Überzug für die Ultraschallsonde, Abdecktücher, Kompressen, Steristrips, Kompressionsbinden ( $\triangleright$ Abb. 1).

Matrial bei Schaumsklerosierung/Exhairese | Zusätzlich $2 \times 10 \mathrm{ml}$ Spritze, Aufziehnadel, Zwischenstück zur Schaumherstellung, Aetoxysklerol ${ }^{\circledR}$, Injektionsnadel. Bei geplanter Exhairese zusätzlich Varadyhaken und mehrere Moskitoklemmen.

Abb. 4 (a) Zeigt der Nadelschliff bei der Punktion nach oben, so ist die Nadelspitze bei Eintritt in die Vene im Ultraschall besser zu erkennen. (b) V. saphena magna mit Führungsdraht, rechts im Bild ist die Spitze der Punktionsnadel zu sehen.
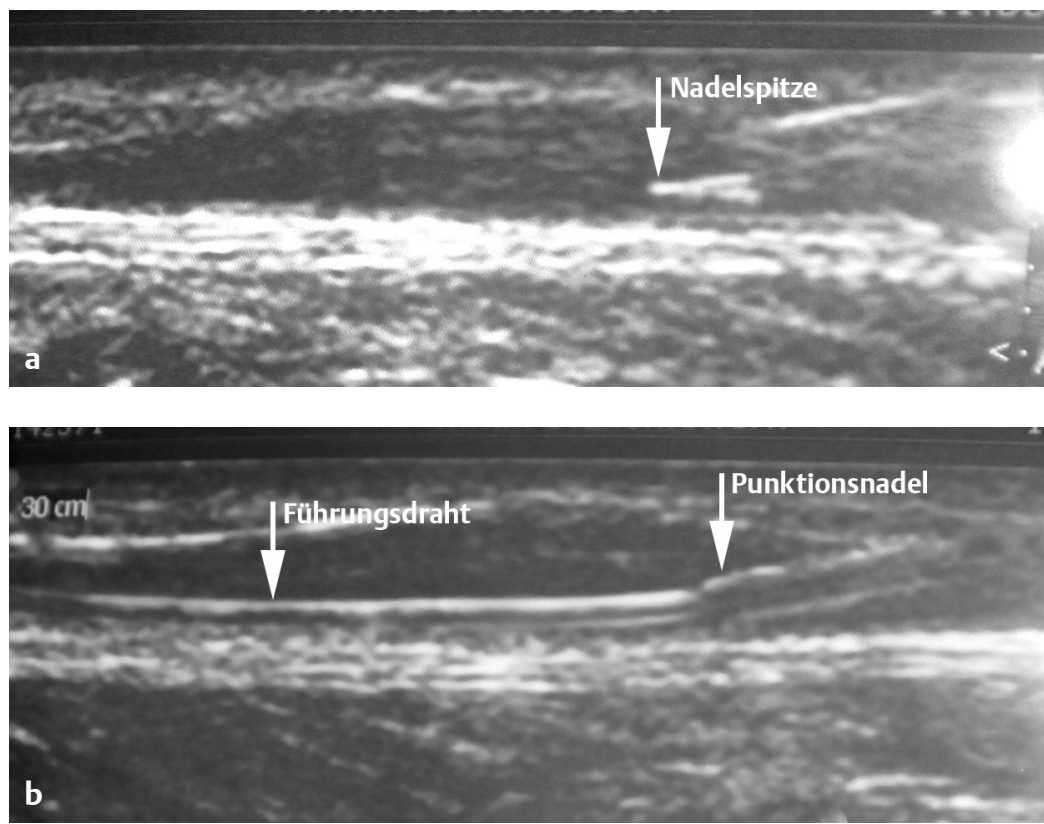


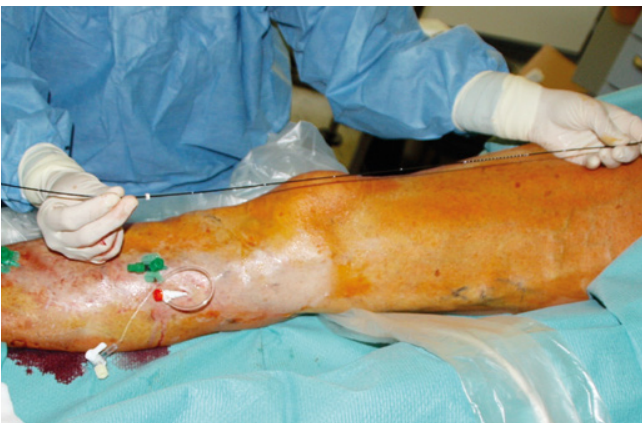

Abb. 5 Vor dem Einführen des Katheters wird die Länge bis zur Crosse abgemessen.

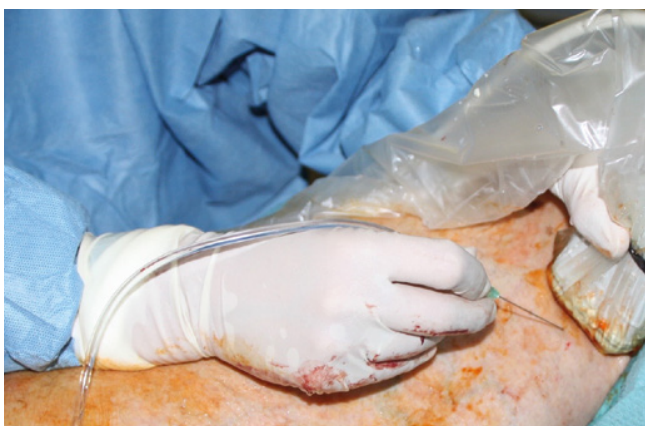

Abb. 7 Tumeszenzanästhesie unter Ultraschallkontrolle in die Faszienloge der V. saphena magna.

\section{Radiofrequenzablation der V. saphena magna}

\section{Schritt-für-Schritt-Anleitung}

1. Duplexsonografie der zu behandelnden Gefäße im Stehen, Anzeichnen des Venenverlaufs und anatomischer Besonderheiten

2. Steriles Abdecken und Desinfizieren, Rasieren im Punktionsbereich, Beintieflage ( Abb. 2)

3. Ultraschallgesteuerte Punktion im Längs$\operatorname{schnitt}(>$ Abb. 3)

Cave Keine Punktion am distalen Unterschenkel: Gefahr einer hitzeinduzierten Nervenläsion!

4. Einführen des Führungsdrahts in SeldingerTechnik ( Abb. 4), Schnitterweiterung, LokalAnästhesie-Quaddel, Einführen der Schleuse (7F), Ultraschallkontrolle

Cave Das Vorschieben des Führungsdrahts und später des Katheters sollte leichtgängig ohne Widerstand sein. Ist dies nicht der Fall, muss durch eine sonografische Kontrolle eine Fehllage oder postphlebitische GefäßwandVeränderungen ausgeschlossen werden.

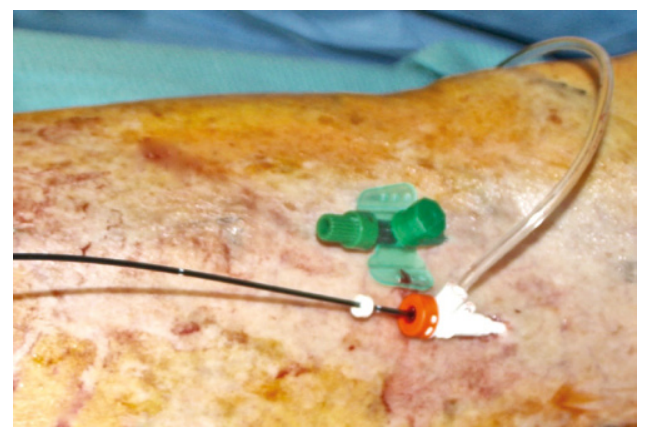

Abb. 6 Die Längenabmessung wird mittels am Katheterschaft angebrachten weißen Kunststoffrädchen fixiert, so dass der Katheter nicht unbeabsichtigt über die Crosse in die tiefe Vene vorgeschoben wird.

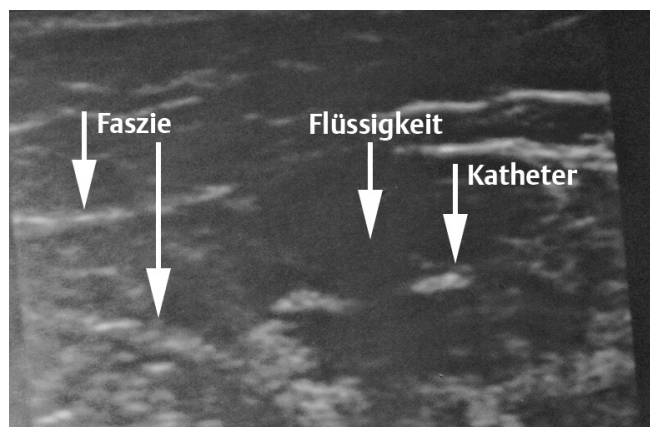

Abb. 8 Faszienloge der V. saphena magna mit Flüssigkeitssaum um den Katheter (Querschnitt).

5. Spülen des Katheters mit Kochsalzlösung, Katheterlänge abmessen ( Abb. 5), Katheterlänge begrenzen mit weißem Kunststoffring am Katheterschaft ( $\triangleright$ Abb. 6 ),

6. Einführen und Vorschieben des Katheters unter Ultraschallkontrolle bis $1-2 \mathrm{~cm}$ vor die Crosse

Cave Eine Positionierung der Katheter-Spitze zu nah am tiefen Venensystem kann zur Thrombus-Extension ins tiefe Venensystem führen.

7. Kopf-Tieflage zur Venenentleerung, ultraschallgesteuerte Tumeszenzanästhesie ( Abb. 7 und 8 )

8. Kontrolle der Katheterlage und gegebenenfalls Repositionierung

9. Impulsabgabe am Handstück des Katheters, manuelle Kompression im behandelten Gefäßabschnitt

10. zweite Impulsabgabe im crossennahen Segment, segmentaler Rückzug des Katheters, abschnittsweise Gefäßbehandlung ( Abb. 9 und 10)

11. Rückzug der Schleuse, letzte Impulsabgabe mit mindestens $2 \mathrm{~cm}$ Hautabstand (Kathetermarkierung)

12. Entfernung des Katheters, manuelle Kompression, Klammerpflaster 


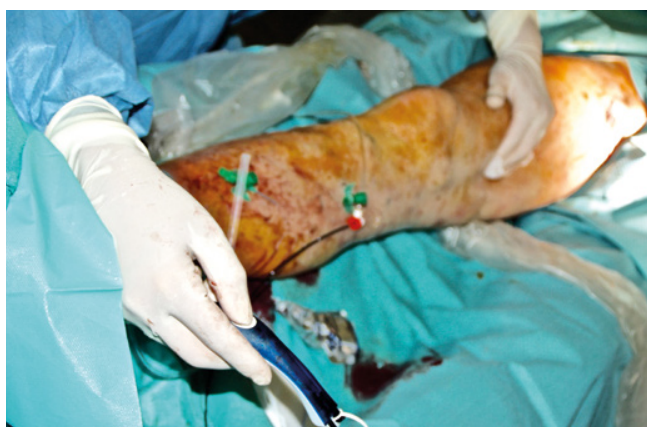

Abb. 9 Mit dem Handstück werden die 20 Sekunden andauernden Heizintervalle ausgelöst. Die externe manuelle Kompression entlang des Gefäßes verbessert den Gefäßandruck

13. Exhairese oder Schaumsklerosierung der Seitenastvarikosis, falls notwendig (siehe unten)

14. steriler Kompressionsverband

Warum Tumeszenzanästhesie? | Die Tumeszenzanästhesie dient der Lokalanästhesie, aber auch der Kompression der zu behandelnden Vene und Schutz des umgebenden Gewebes vor hitzeinduzierter Schädigung. Eine Kühlung des TumeszenzLösung vor der Applikation erhöht den Schutzeffekt. Die Applikation erfolgt sonografiegestützt paravasal in die Faszienloge (,saphenous compartment“) [7].

\section{Zusammensetzung Tumeszenzanästhesie I}

- $1000 \mathrm{ml}$ isotone Kochsalzlösung 0,9\% mit $50 \mathrm{ml}$ Prilocain 1\%, 6-12 ml Natriumhydrogencarbonat $8,4 \%, 1 \mathrm{ml}$ Epinephrin 1:1000.

Die Beigabe von Epinephrin dient der Wirkungsverstärkung der Tumeszenanästhesie und Reduzierung der Blutungsneigung. Sie ist nach Expertenmeinung jedoch auch verzichtbar, um kardiale Nebenwirkungen zu vermeiden [1].

\section{Schaumsklerosierung der Seitenastvarikosis}

Sklerosierungsmittel | Aetoxysklerol ${ }^{\circledR}$ mit dem Wirkstoff Polidocanol ist das einzige in Deutschland zugelassene Sklerosierungsmittel. Die Injektion der gewebetoxischen Flüssigkeit in eine Varize führt zu einem lokalen Endothelschaden und zum Gefäßverschluss (Verödung). Aetoxysklerol ${ }^{\circledR}$ steht in verschiedenen Dosierungen zur Verfügung (0,25-3\%). Generell gilt: Je größer der Gefäß-Durchmesser, desto höher die gewählte Dosierung, d.h. 0,5\% für Besenreiser und retikuläre Varikosis, 1-2\% für Seitenastvarikosis, 3\% für Stammvenen. Der Sklerosierungsschaum wird durch Mischung von Aetoxysklerol ${ }^{\circledR}$ mit Raumluft im Verhältnis 1:4 hergestellt und mittels Doppelspritzentechnik (DSS) aufgeschäumt. Die maximale Dosis ist $2 \mathrm{mg}$ Polidocanol pro kg Körpergewicht. Bei der Schaumsklerosierung sollte allerdings in der Regel eine maximale Applikati-

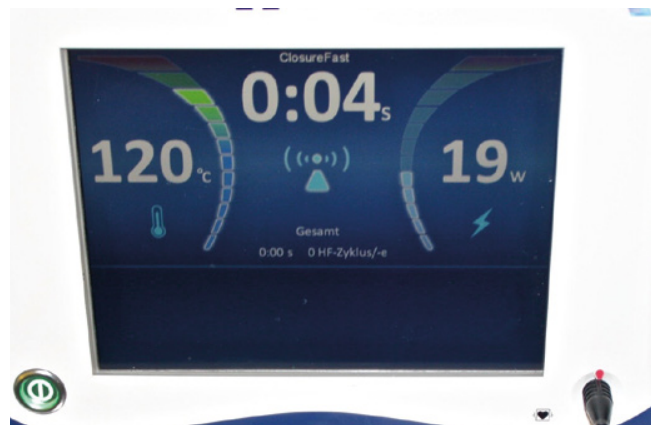

Abb. 10 Der Generator zeigt die erreichte Temperatur am Heizelement, die Sekundenzahl des Impulses sowie die Wattzahl an. Hier erfolgte noch eine zweite Impulsabgabe

on von $10 \mathrm{ml}$ Schaum in einer Sitzung nicht überschritten werden $[5,6]$.

Ablauf

1. Darstellung der Seitenastvarikosis im Ultraschall

2. größere, tiefliegende Seitenastvarikosis: Punktion unter Ultraschallsicht (quer)

$(\triangleright$ Abb. 11)

> oberflächlichere Anteile:

Punktion mit Hilfe einer Kaltlichtquelle (Veinlite ${ }^{\circledR}$; s.u.) ( Abb. 12)

3. Kontrolle der intravasalen Lage

4. Injektion des unmittelbar vorher angemischten Sklerosierungsschaums ( $\triangleright$ Abb. 13 und 14)

5. exzentrischer Kompressionsverband, Kompressionstherapie bis zu 2 Wochen tagsüber

6. sonografische Ergebniskontrolle, Stichinzision und Thrombusexpression bei der Nachkontrolle nach Bedarf

Kaltlichtquelle | Die Kaltlichtquelle produziert mit orangenen und roten LEDs einen nach unten und in die Ringmitte gerichteten Lichtstrahl, der das Gewebe einige Millimeter tief erleuchtet (Transillumination). Die oberflächlich gelegenen Venen werden als dunkle Strukturen im umliegenden Gewebe sichtbar und können für die Sklerotherapie über die Ringöffnung anpunktiert werden.

\section{Nach dem Eingriff}

Überwachung I An den Eingriff schließt sich eine kurze Überwachungszeit an, der Patient kann in der Regel ca. eine Stunde nach dem Eingriff entlassen werden.

Thromboseprophylaxe | Wir führen über 5 Tage eine Thromboseprophylaxe mit Tinzaparin (Innohep $\left.^{\circledR}\right) 3500$ IE/die durch. Bei bekannter Thrombophilie oder Hochrisikopatienten kann die Prophylaxe entsprechend erweitert werden, zum Beispiel mit Enoxaparin (Clexane ${ }^{\circledR}$ ) $0,4 \mathrm{ml}$ über 10 Tage. 


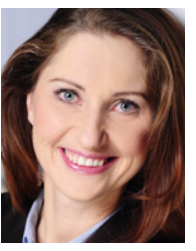

Dr. med. Anina Lukhaup Innere Medizin und Angiologie in der Gefäßpraxis im Tal in München. lukhaup@angiopraxis.de arbeitet als Fachärztin für

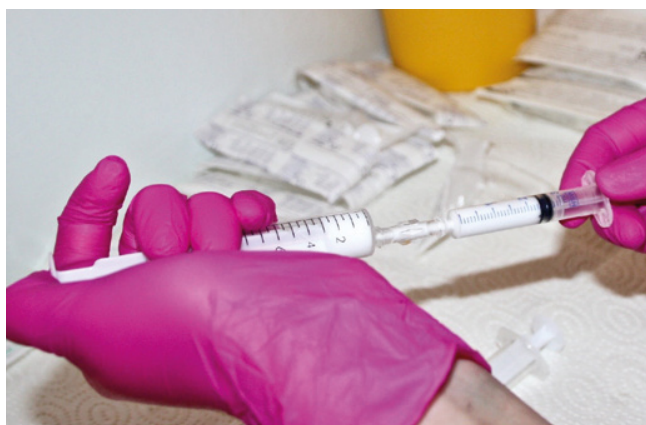

Abb. 13 Der Sklerosierungsschaum wird unmittelbar vor der Applikation aufgeschäumt.

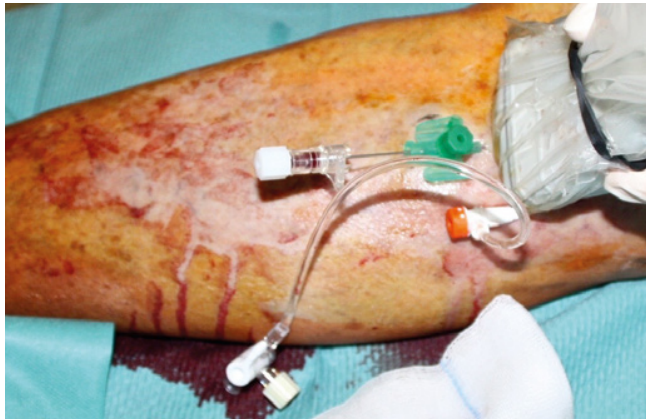

Abb. 11 Unter Ultraschallsicht erfolgt die Punktion der Seitenäste.

Kompressionsbehandlung | Die Kompressionsbehandlung erfolgt regelhaft über 14 Tage mit oberschenkellangen Kompressionstrümpfen Klasse II. Eine Verkürzung der Kompressionsdauer oder Reduzierung des Kompressionsdrucks (Klasse I) ist in Absprache mit dem Patienten möglich.

Nachkontrolle I Nachfolgende farbduplexsonografische und klinische Kontrollen nach 7-10 Tagen und drei Monaten dienen dem Ausschluss von Komplikationen (insbesondere endovenöse hitzeinduzierte Thrombose=EHIT) und der Detektion von notwendigen Nachbehandlungen. Nach abgeschlossener Behandlung empfehlen wir Kontrolluntersuchungen im jährlichen $\mathrm{Ab}$ stand.

Nachbehandlung I Im Falle einer inkompletten Rückbildung der Seitenastvarikosis durch Ausschaltung der Stammveneninsuffizienz erfolgt ca. 3 Monate nach dem Eingriff eine erneute Schaumsklerosierung

\section{Literatur}

1 Alm J, Böhme J, Kensy M. Entwicklung der VNUS-Radiofrequenzkatheter-Therapie in der Behandlung der Varikose. Phlebologie 2010; 39: 61-68

Dtsch Med Wochenschr 2016; 141: 1316-1320

(c) Georg Thieme Verlag KG . Stuttgart . New York. ISSN 0012-0472

2 S2-Leitlinien Diagnostik und Therapie der Krampfaderbehandlung der DGP, DGG, Berufsverband der Phlebologen

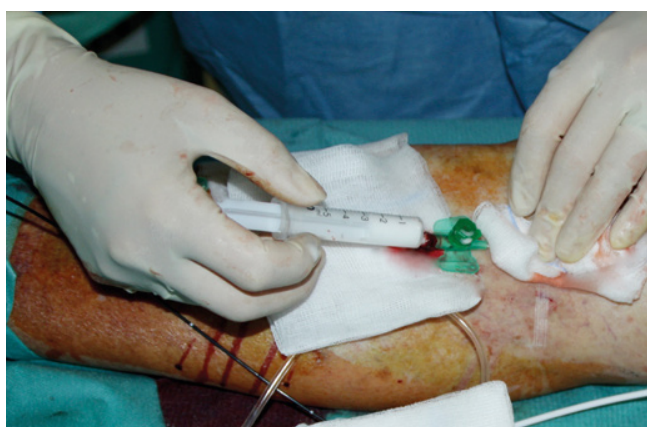

Abb. 14 Nach Applikation des Sklerosierungsschaums wird die Venenverweilkanüle entfernt und komprimiert.

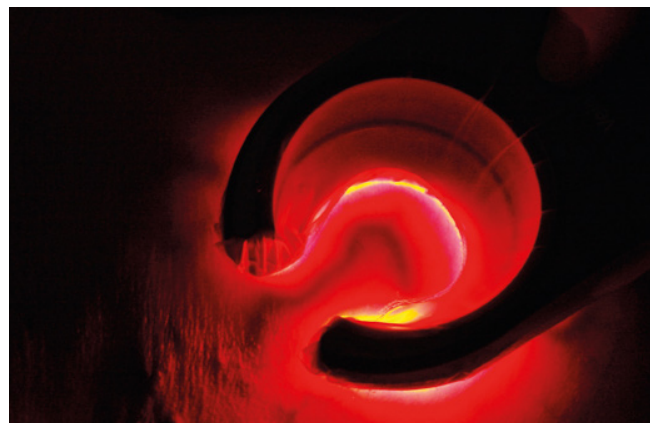

Abb. 12 Die Kaltlichtquelle macht oberflächlichere Gefäßanteile sehr gut sichtbar und eignet sich auch zur kosmetischen Behandlung der retikulären und Besenreiservarikosis.

\section{Konsequenz für Klinik und Praxis}

- Die segmentale Radiofrequenzablation ist eine schonende ambulante Behandlung der Stamm- und Seitenastvarikosis.

- Die Behandlung einer beidseitigen Varikosis ist in einer Sitzung möglich.

- Die Methode ist unter Antikoagulation durchführbar.

- Für die Patienten bedeutet die Behandlung Keine oder nur kurzzeitige Arbeitsunfähigkeit und Einschränkung der Alltags- und sportlichen Aktivitäten.

- Eine fachgerechte Indikationsstellung ist wichtig: Nicht jede asymptomatische Varikosis gehört operiert [1].

- Die Kosten werden von den privaten und inzwischen auch von vielen gesetzliche Krankenkassen (zum Teil über extra abzuschließende Verträge) übernommen.

- Es stehen verschiedene endovenöse Therapieverfahren zur Auswahl.

3 Deutsche Gesellschaft für Phlebologie. www. phlebology.de (Letzter Zugriff: 15.7.2016)

4 Hartmann K. Endovenöse Verfahren. Minimalinvasive Therapie der Varikosis. Stuttgart: Schattauer; 2015 
5 Hübner K, Breu X. Praktische Sklerotherapie - Sklerosierungsbehandlung der Varikose und anderer Indikationen. Köln: ViaVital; 2013

6 Deutsche Gesellschaft für Phlebologie. S1-Leitlinien Sklerotherapie. http://www.awmf.org/uploads/ tx_szleitlinien/037-015I_S1_Sklerosierungsbehandlung_Varikose_2015-01.pdf (Letzter Zugriff 18.7.2016)

7 Mendoza E. Duplexsonographie der oberflächlichen Beinvenen. Berlin: Springer; 2013 